\title{
Identification of SARS-CoV-2 preclinical (in vivo) compounds targeting COVID-19 main protease: A scoping review, meta-analysis and molecular docking studies
}

Oluwakemi Ebenezer

Mangosuthu University of Technology

Maryam Amra Jordaan ( $\square$ maryamamrajordaan@gmail.com )

Mangosuthu University of Technology https://orcid.org/0000-0002-3057-123X

Nkululeko Damoyi

Mangosuthu University of Technology

Ropo Ogunsakin

University of KwaZulu Natal

Michael Shapi

Mangosuthu University of Technology

\section{Research Article}

Keywords: COVID-19, meta-analysis, molecular docking, pre-clinical, SAR-CoV

Posted Date: May 22nd, 2020

DOI: https://doi.org/10.21203/rs.3.rs-31140/v1

License: (c) (1) This work is licensed under a Creative Commons Attribution 4.0 International License.

Read Full License 


\section{Abstract}

Due to the migratory flow of infected people with severe acute respiratory syndrome virus (SARS-CoV-2), the numbers of confirmed cases of coronavirus 2019 (COVID-19) infections is accelerating worldwide and pre-clinical evidence of antiviral agents that can combat this pandemic is still elusive. We identified published SAR-CoV efficacy experiments in which some selected compounds were used to test the reduction of the virus load in mice. We then developed a combined model based on scoping review, metaanalyses, and molecular docking studies to evaluate the effect size of preclinical studies of compounds that have been tested against SARS-CoV. Molecular docking studies of the inhibitors in the active pocket of COVID-19 protease were also performed. Our results identified three SARS-CoV inhibitors i.e. EIDD2801, GS-5734 and amodiaquine that are excellent options for optimization and drug development to treat or cure COVID-19.

\section{Introduction}

The coronavirus disease that originated in 2019 (also referred to as COVID-19) is an emerging infectious disease that is caused by severe acute respiratory syndrome coronavirus 2 (SARS-CoV-2). The global pandemic disease which sprung forth from Wuhan City, in Hubei Province of China was first reported by the World Health Organization (WHO) on 31 December 2019. Globally, the recent estimate of SARS-CoV-2 as of April 7, 2020, indicated that $\sim 1360$ million people are infected and $\sim 75,933$ thousands of deaths have been recorded. Meanwhile, the migratory flow of infected people has accelerated the spread of the virus, especially in the United State of America (363,376 cases) and Spain (136,675 cases) and this had led to mandatory isolation or quarantine of people across the globe. Moreover, the outbreak of COVID-19 infection is not effectively controlled due to its strong capacity of transmission: (i) human to human i.e., close proximity with infected people via respiratory droplets from cough or sneezing, (ii) touching the surface or object contaminated with the virus and later on touching their mouth, nose, or eyes (iii) aerosol transmission [1-5]. Consequently, the aftermath could potentially threaten worldwide health systems and have a negative influence on the global economy [6]. Heshui et al. [5] noted that elderly people and those that have an underlying illness such as chronic pulmonary disease and diabetes are more susceptible to COVID 19 infection [5]. In another study, Ying et al. reported that many patients with underlying cardiovascular diseases (CVDs) might have an increased risk of death [7]. Additionally, the expert consensus statement released by Yen et al, reviewed that no positive cases of newborns have been reported yet, and infected children show symptoms such as; fever, dry cough, and fatigue, and few have upper respiratory tract infection [8]. Due to a rapid increase in confirmed cases, COVID-19 has been considered to be a menace to humankind, hence, an urgent need for drastic measures is therefore required to design and develop an antiviral agent that can target COVID-19, so that treatment can be timely, expeditious, while reducing the mortality rate [7].

Severe acute respiratory syndrome virus (SARS-CoV) and Middle East respiratory syndrome virus (MERS$\mathrm{CoV}$ ) are in the same class of betacoronavirus alongside SARS-CoV-2. SARS-CoV main proteinase, $3 \mathrm{CLpro}$, which is also referred to as $\mathrm{M}^{\text {pro }}$, is a homodimer with three structural domains: domain I, II and 
III and highly conserved among SARS-CoV and MERS-CoV, displaying 40\% - 44\% sequence homology (12). Whereas, 3CLpro protease of SARS-CoV-2 share over $95 \%$ of sequence similarity with those of the SARS-CoV despite, the fact that these two viruses demonstrated only $79 \%$ sequence similarity at the genome level; as well as the similar active site $[6,9,10]$. In addition, 3 CLpro plays a crucial role as functional intermediates involved in the replication and transcription of viral RNA, which makes it turn-out as the most potential attractive anti-coronavirus target $[11,12]$.

Unfortunately, at present, there is neither a vaccine nor drug regime to combat COVID-19. This has caused a global race for researchers and scientists to develop, an effective, bioavailable antiviral agent with minimal side effects to fight COVID-19. To support the ongoing research and development efforts to discover effective agents that can target coronavirus, we did a scoping review of preclinical (in vivo) evidence on selected compounds against SARS-CoV (Fig.1) [6]. Furthermore, meta-analyses were used to calculate the effect size by exploring the main characteristics of the experimental models, virus strains and the lung virus titer, since clinical decisions should be based on the totality of the best evidence and not on the results of individual studies. The rationale for the meta-analyses and in vivo experiment, molecular docking studies were performed to analyze and obtain SARS-CoV inhibitors that can be optimized to combat COVID-19.

\section{Methods}

2.1 Search Strategies. The search strategy was based on the methodological framework suggested by Moher et al., namely, Preferred Reporting Items for Scoping Review and Meta-Analysis (PRISMA) [13]. The meta-analysis of preclinical evidence of SAR-CoV inhibitors was initiated with focus incline keywords using search words: 'SARS-CoV', 'SARS', 'COVID-19 OR COVID-19 infection', 'coronavirus', 'severe acute respiratory syndrome virus', OR 'in vivo,' 'SARS-CoV main protease', 'protease', 'preclinical evidence', 'animal model', mice, 'SAR-CoV inhibitors', 'mouse', 'BALB/c'. The search was carried out in three electronic databases: PubMed, Web of Science and Scopus. The entire databases were searched until April 5, 2020 and all relevant studies were recovered and included in the scoping review and metaanalysis. The criteria 'filtered results' was applied to exclude conference proceedings, review article and book chapters.

2.2 Study Selection and Eligibility Criteria. In the scoping review and meta-analysis, we included published studies that are preclinical (in vivo) evidence of SARS-CoV agents, regardless of the country. The included studies were published in an English language reputable journal. Since the number of articles on preclinical (in vivo) evidence on the SARS-CoV agent is relatively small, no restrictions were made for the dates of publication.

2.3 Selections of the Studies. The study selection process is shown in Fig. 2. All citations $(N=225)$ identified through our search strategy were imported into EndNote version X7 reference management software and used automated "Find Duplicates" function to exclude any duplicates. The title and abstracts of the 225 articles were assessed by two reviewers (OE \& RO). 
2.4 Data Extraction. A standardized and pre-piloted checklist was used to extract the required information. Data was extracted on (i) publication characteristics, (ii) experimental design (animal models and disease models): species, linage, sex, age, and weight, virus species and strain, route of inhibitors administration and (iii) research outcomes (virus titer, histopathological, immunological maker, findings and mortality). Only the outcomes of the final tests were included in case they were performed at different points, and only the data of the highest dose of the inhibitors in the experimental group of animals were included.

Mean and standard deviation (mean SD) were directly extracted when available in the tables or text and values reported in graphics were extracted with image analysis software program (Image-Pro Plus 4.5, Media Cybernetics, Bethesda, Maryland, USA)

2.5 Data Analysis and Synthesis. The data extracted were captured into an excel spreadsheet and later imported to STATA for further analysis. An important statistical issue in meta-analysis is the handling of heterogeneity among studies. We evaluated heterogeneity with DerSimonian and Laird $P$ statistics for each analysis category [14]. The visual assessment of publication bias was assessed by funnel plots. A Begg-adjusted rank correlation test was also used to check for publication bias. If these tests showed non-significant heterogeneity, we used a fixed-effects model, otherwise, a more conservative randomeffects model was used [15]. We assessed heterogeneity between studies using Cochran's $Q$ statistic and the $R$ statistic $[14,16]$, which estimated the percentage of total variation across studies. $P$ statistics lie between 0 to 100 percent, of which, a value of $0 \%$ implies no heterogeneity while $100 \%$ indicates the presence of significant heterogeneity. A sensitivity analysis was performed to assess the influence of each study. Data manipulation and all statistical analyses were done using Stata/IC software, version 15.0.

2.6. Molecular Docking. Molecular docking analysis was performed to explore the binding pattern of the SARS-CoV agents into the binding site of COVID-19 main protease. The X-ray crystal structure of COVID19 main protease (PDB ID: 6lu7) was retrieved from RCSB Protein Data Bank [17]. Using Autodock vina, the ligand and receptor complexes were prepared for docking. Chem3D Ultra was used to construct a three-dimensional structure for all the compounds in the study, followed by structure optimization. AutoDock Tools ( $V$ 1.5.6) were used to establish grid box parameters, all non-polar hydrogens were merged, Gasteiger partial atomic charges added, and rotatable bonds assigned [18]. The grid box of the complex had a grid spacing of $0.375 \AA$. The grid center and grid size were set: $X=40 /-20, Y=31 / 13, Z=$ $65 / 47 \AA$. Ligand-receptor interaction of the ligands was thereafter simulated into the binding site of COVID-19 main protease. The resulting docking studies were validated by redocking the native ligand of the protein crystal structures into the binding site. Analysis and visualization of binding interactions in the protein-ligand complex were achieved using Discovery Studio Visualizer.

\section{Results}

The literature search strategy included 225 articles. After removing the duplicates, 190 were excluded just by reading their titles. Of the remaining studies, 4 were excluded based on the abstract and the outcome 
variable. Hence, the remained 6 studies were included in the final analysis (Figure 2).

3.1 Characteristics features of the included studies: A total of six studies were included in scoping review and meta-analysis [19-24]. All studies originated from the United States of America (USA) and utilized mice and marmosets, but only the analyses with mice are taken into consideration. Besides, none of the studies reported the approach used to arrive at the determination of the sample size calculation utilized and the order in which the groups were treated. For the allocation of the administration usage: intraperitoneal $(n=2,33.3 \%)$; intraperitoneal and intranasal $(n=2,33.3 \%)$; oral $(n=1,16.6 \%)$ and subcutaneous $(n=1,16.7 \%)$.

3.2 Features of the animal model: As reported above, all the studies utilized mice as the animal model. The linage is distributed as follows: BALB/C ( $n=4,66.7 \%)$; C57B46J ( $n=1,16.7 \%)$ and both C57B46J and $\mathrm{Ces} /{ }^{-/-}(n=1,16.7 \%)$. Four of the studies $(n=4)$ used female animal and it represents $66.7 \%$ of the entire studies while 33.3\% ( $n=2)$ of the studies used animals of both sexes. None of the studies utilized only male animals. The animals' age was reported in weeks, of which each category represents about $17 \%$ each and three of the studies did not report the age of their animal. Meanwhile, the animal weight raged from 11-18g, such that animals with weight $11-16 \mathrm{~g}$ had the highest proportion $(\mathrm{n}=2,33.3 \%)$ while two of the studies did not report the weight of the mice. Urbani $(n=3,50.0 \%) ; V 2163(n=1,16.7 \%)$ and MA15 ( $n=2,33.3 \%$ ) were the SARS-CoV strains finally used for in vivo experiment. (Table S1)

3.3 Meta-analysis result: We use the standardized mean difference (SMD) for this meta-analysis. An SMD is adopted regularly in clinical meta-analyses due to its ability to express the difference between the groups relative to the standard deviation. It can be seen from the result that the test of heterogeneity yields $Q=103.57$, degree of freedom $(\mathrm{df})=15$, resulting in a $p$-value $<0.0001$. This indicates statistically significant heterogeneity among the sixteen compounds which lead to the random-effects model. Using the random-effects model, the combined SMD $=0.781$ with $95 \%(0.147,1.595)$ with $p$-value $<0.001$, indicating a statistically significant difference between the compounds and control (placebo or vehicle) in terms of virus titer. Eleven out of the fifteen compounds showed a significant reduction in virus titer, indicating an effect in favour of treated animals compared to placebo (Fig. 3).

Subgroup analysis by study compounds was conducted to assess the potential heterogeneity between studies. Out of the two therapies, the highest estimated of mortality was found in studies with prophylactic (21.8\% (95\% Cl: $16.4 \%$ to $28.8 \%), P=64.2 \%)$, followed by studies conducted with therapeutic, was $10.6 \%$ (95\% Cl: $14.2 \%, 22.7 \%), R=0.0 \%$ (Fig. 4). Hence, the overall mortality was reduced in mice treated with the compounds (RR: 1.80 [95\% Cl: 1.42, 2.27], $P=0.020$ ).

\section{Discussion}

SARS-CoV emerged from a zoonotic reservoir and couple with cytokine, chemokine, and Interferon Stimulated Gene (ISG) responses in patients provided evidence that SARS-CoV pathogenesis is partially controlled by innate immune signaling [25]. Dysregulation of inflammatory cytokines such as tumor 
necrosis factor (TNF)- $a$, interleukin (IL)-1 $\beta$, IL-6, IL-10] and polymorphonuclear neutrophil (PMN); and chemokine IL-8 are responsible for the indication of viral infection, which may be due to activation of transcription factor nuclear factor (NF)- $k$ B, activator protein (AP)-1 and activating factor-2 (ATF-2) [2629]. This is also observed in SARS-CoV-2. Thus, identifying specific molecules to destroy the immune evasion of SARS-CoV-2 is very crucial.

Scoping or systematic reviews of preclinical have served as a robust form of knowledge synthesis to evaluate transparently experimental therapies for more than a decade [30]. Our findings from the metaanalysis indicated the effects of the selected compounds in the reduction of the virus titer. The treatment efficacy is represented by the midpoint and the length of each segment in the forest plot while the diamond shape showed the combined effect (Figure. 3). Further, results from meta-analysis, in vivo and molecular docking studies corroborate three compounds, which are EIDD-2801, GS-5734 and amodiaquine. The histopathology and immunological findings of all the compounds against the SARSCoV virus are shown in Table 1.

The understanding of the similarity between the 3CLpro main protease of SAR-CoV and COVID-19 which is very important in the viral life cycle has highlighted the protease as an attractive potential target for anti-coronavirus drug development [31-33]. In addition, they both have catalytic dyad composed of conserved residues His41 and Cys145. Considering the in vivo activity, of the investigated compounds, their molecular affinity in the binding pocket of COVID-19 main protease was evaluated. Only, seven out of the compounds showed good binding energy. They bind with COVID-19 protease via multiple hydrogen bonding and hydrophobic contacts. Residues Phe140, 185 Leu141, His163, Met165, Glu166, and His172 form hydrophobic interactions, whilst Asn142, Gly143, and Cys 145 form intermolecular H-bond with the ligands. The binding mode indicates useful clues to the possible molecular basis of these compounds.

Table 1: Results obtained in infected mice treated with the selected compounds [19-24] 


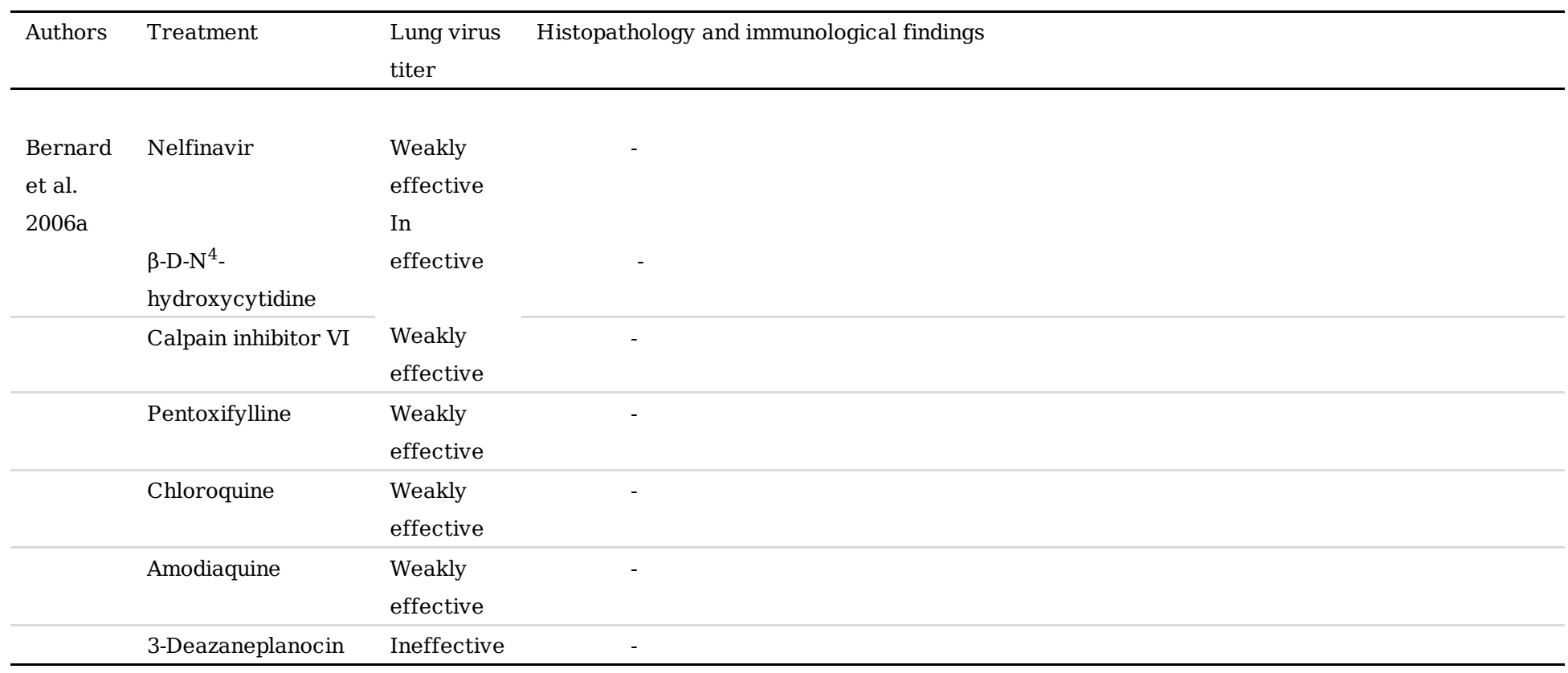

Mycophenolic acid Ineffective Increase in viral replication

Bernard

et al.

$2006 \mathrm{~b}$

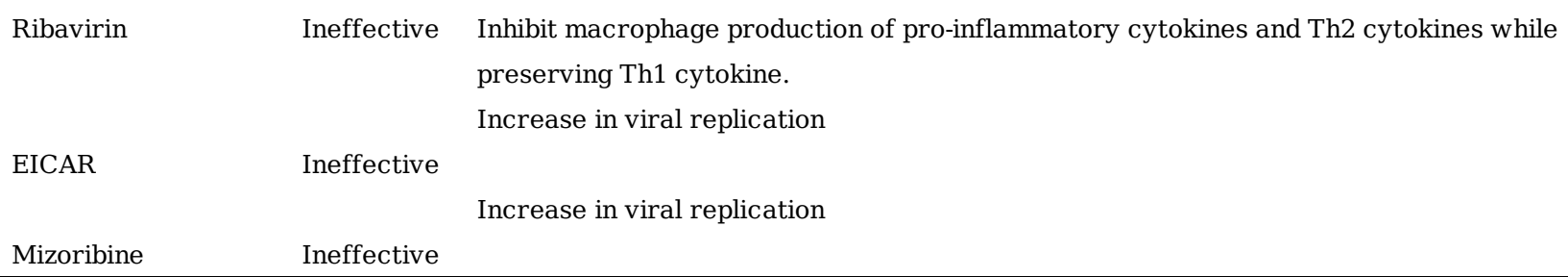

Ribavirin Weakly

Allowed continual stimulation of the inflammatory response, which may contribute to effective

Craig

et.al

2009

Dipeptidyl

glutaminyl

fluoromethylketone

(EP128533)

Sheahan Remdesivir (GS- Effective
Ineffective Neither effective in preventing death nor reduced the disease signs measured. No evidence of toxicity and lack of activity may be likely due to bioavailability. May be a foundation for development of an effective antiviral prodrug that is soluble and bioavailable
Therapeutic administration of

GS-5734 reduced disease and suppressed replication of SARS-COV during an ongoing infection. Improve pulmonary function, reduce viral loads, with no development in disease outcomes, thus not clinically beneficial.
Sheahan $\quad \beta-D-N^{4}-$

et al. hydroxycytidine-5'

2020 isopropyl ester

(EIDD-2801) 
As shown in Figure 5, the oxygen atom of the primary hydroxyl functional group of nucleoside unit EIDD1931 (B.E $=6.5 \mathrm{kcal} / \mathrm{mol}$ ) is $\mathrm{H}$-bonded to Cys 145 with bond distance of $2.59 \AA$ and bond angle of 127.7 and $100.5^{\circ}$, respectively, while, the hydrogen atom is H-bonded Ser144 and Le141 (bond distance $=2.73$ and $2.68 \AA$; bond angle $=129.1,114.7,127.7$ and $100.5^{\circ}$, respectively). The conserved amino acid His41 is sandwiched between the pyrimidinone ring and the hydroxy amino group via $\mathrm{H}$-bonding and hydrophobic interaction.

The interactions of 3-Deazaneplanocin (B.E $=6.5 \mathrm{kcal} / \mathrm{mol}$ ) with the COVID-19 main protease is described in detail in Figure 2. Interestingly, the cyclopentene unit of this compound interacts with Cys145 via Hbonding, whereas, an oxygen atom of the primary hydroxy functional group on cyclopentene ring forms three notable hydrogen bonds with a backbone amino acid residue Gly143 and 145 having the bond distance of 2.59 and $2.25 \AA$ respectively; the hydrogen atom formed $\mathrm{H}$-bond interactions with Ser144 and Leu 141, these interactions were also observed in the docked complex of EP128533. Meanwhile, the amino acid Thr26 is interpolated in between $\mathrm{NH}_{2}$ and $\mathrm{NH}$ functional group of imidazo[4,5-c]pyridine moiety. (Figure. 6)

The oxygen atoms on the carbonyl group of fluoromethyl ketone and dimethylamine (EP128533, B.E = $6.9 \mathrm{kcal} / \mathrm{mol}$ ), dimethylammine forms a H-bond with the side-chain nitrogen of Gly143 and Thr26, while the $F$ atom interacts with Cys145 and Gly143 via another H-bond. The benzyl group in the unit formed interaction with the sulphur of Met165. In addition, the carbamate group fits into a small hydrophobic pocket, which is favorable for $\pi-\sigma$ and alkyl interaction between the methylene as well as methyl group and amino acid His41 and Met49 respectively, whereas the amine group H-bonded with $\mathrm{Gln} 189$. The increase in the interaction of the inhibitor in the active pocket of COVID-19 protease can be rationalized to be the reason for the increase in the binding affinity of the inhibitor. (Figure.7)

The landscape of amodiaquine docked complex (Fig. 8) was similar to that of EIDD-1931, in the sense that the chloroquinoline group was inserted in the hydrophobic pocket consisting of Met165 and His41. The amino acid Cys145 was H-bonded to the benzyl ring as well as the oxygen atom of the tertiary hydroxyl functional group in the unit. Besides, the hydrogen atom formed $\mathrm{H}$-bond as shown in Figure 4: the first $\mathrm{H}$-bond is from Leu141 (bond distance $=1.90 \AA$ ), the second is from Ser144 (bond distance = $2.53 \AA$ ). The presence of these two interactions in the binding pocket helps in positioning and stabilizing the docked complex of amodiaquine.

Furthermore, the interactions of nelfinavir (B.E $=8.2 \mathrm{kcal} / \mathrm{mol})$ in the binding pocket of COVID-19 protease are shown in Figure 9. The phenylthiol was deeply inserted within the hydrophobic pocket and interacts to formed: $\pi-\pi-T$ stacking contact with amino acid His41, $\pi$ - alkyl with Met49 and $\pi$-sulpur with Met165. Moreover, the $-\mathrm{CH}_{3}$ group on hydroxymethylbenzamide interact with His41 and Leu27 via hydrophobic, while the carbonyl oxygen atom involved in the H-bond network with Gly143. Moreover, Asn 142 formed Hbonding with an amine and hydroxyl functional group of isoquinoline carboxamide. It was noted that 
nelflavir has the highest binding affinity $\left(8.2 \mathrm{kcal} \cdot \mathrm{mol}^{-1}\right)$ which correlates with its highest interaction in the binding pocket of COVID-19 protease, hence confirm its highest stabilization compared to other inhibitors but this lack correlation with meta-analysis and in vivo result, hence the modification of this compound may effectively reduce the virus replication. (Figure. 9)

Interestingly, the predicted binding mode of remdesivir (B.E $=7.5 \mathrm{kcal} / \mathrm{mol})$ in Figure 10 showed interaction with the catalytic dyad. The phenyl moiety formed $\pi$-sulphur interaction with catalytic amino acid His41 and $\pi$-cation with Met49. All the amines group formed H-bond with amino acid Thr45, Thr25 and $\operatorname{Gln} 149$ (b.d = 1.84, 2.34 and $2.53 \AA$ respectively), hydroxyl functional group which is in close proximity with Cys 145 accept $\mathrm{H}$-bond from Thr26 (2.38 $\AA$ ). In addition, the methyl group of methyl pentyl acetate formed hydrophobic interaction with imidazoles of His163 and His172.

$\beta$-D-N $N^{4}$-hydroxycytidine-5'-isopropyl ester (EIDD-2801), which is also a nucleoside analog, not only showed high binding but also better interaction compared to $\beta-D-N^{4}$-hydroxycytidine. Interestingly, the carbonyl oxygen of EIDD-2801(B.E $=7.2 \mathrm{kcal} / \mathrm{mol}$ ) accepts H-bond from the backbone of amino acid Gly143, Cys 145, and Ser144. The presence of ester in this compound rationalized the increased inhibitory potency of this compound against SARS-CoV-2. The amino acid, Thr190 was H-bonded with hydroxylamine group, meanwhile, the pyrimidinone ring formed hydrophobic interaction with Met165. Further, the oxygen atom in the nucleoside ring, as well as the primary hydroxy functional group interacts with Glu166 and Asn142 via H-bonding. (Figure. 10).

\section{Conclusion}

Scoping reviews and meta-analyses are essential to summarize evidence relative to drug development, efficacy, and safety of health care interventions accurately. Our meta-analysis showed the overall effect in the reduction of virus titer and mortality in SARS-CoV infected animals. The results from meta-analysis, in vivo and molecular docking studies substantiated three compounds, which are EIDD-2801, GS-5734 and amodiaquine, other SAR-CoV inhibitors that were identified from the molecular docking studies include; Nelflavir, 3-Deazaneplanocin, EP128533 and EIDD-1931. Furthermore, optimization of these compounds as a molecular scaffold to synthesize more active structures using molecular synthesis or library screening of these compounds could be a reasonable strategy or approach in the development of new antiviral agents that targets COVID-19 infection.

\section{Declarations}

Competing interest: The authors declare no competing interests.

\section{References}

1. Li, Q., et al., Early transmission dynamics in Wuhan, China, of novel coronavirus-infected pneumonia. New England Journal of Medicine, 2020. 
2. Adhikari, S.P., et al., Epidemiology, causes, clinical manifestation and diagnosis, prevention and control of coronavirus disease (COVID-19) during the early outbreak period: a scoping review. Infectious diseases of poverty, 2020. 9(1): p. 1-12.

3. https://www.who.int/publications-detail/modes-of-transmission-of-virus-causing-covid-19implications-for-ipc-precaution-recommendations.

4. Meng, L., F. Hua, and Z. Bian, Coronavirus disease 2019 (COVID-19): emerging and future challenges for dental and oral medicine. Journal of Dental Research, 2020: p. 0022034520914246.

5. Shi, H., et al., Radiological findings from 81 patients with COVID-19 pneumonia in Wuhan, China: a descriptive study. The Lancet Infectious Diseases, 2020.

6. Liu, C., et al., Research and development on therapeutic agents and vaccines for COVID-19 and related human coronavirus diseases. 2020, ACS Publications.

7. Zheng, Y.-Y., et al., COVID-19 and the cardiovascular system. Nature Reviews Cardiology, 2020: p. 1-2.

8. Shen, K., et al., Diagnosis, treatment, and prevention of 2019 novel coronavirus infection in children: experts' consensus statement. World Journal of Pediatrics, 2020: p. 1-9.

9. Ton, A.-T., et al., Rapid Identification of Potential Inhibitors of SARS-CoV-2 Main Protease by Deep Docking of 1.3 Billion Compounds. Molecular Informatics, 2020.

10. Zhang, L., et al., Crystal structure of SARS-CoV-2 main protease provides a basis for design of improved a-ketoamide inhibitors. Science, 2020.

11. Nukoolkarn, V., et al., Molecular dynamic simulations analysis of ritronavir and lopinavir as SARSCoV 3CLpro inhibitors. Journal of theoretical biology, 2008. 254(4): p. 861-867.

12. Fang, S.G., et al., Proteolytic processing of polyproteins 1a and 1ab between non-structural proteins 10 and 11/12 of Coronavirus infectious bronchitis virus is dispensable for viral replication in cultured cells. Virology, 2008. 379(2): p. 175-180.

13. Moher, D., et al., Preferred reporting items for systematic reviews and meta-analyses: the PRISMA statement. Int J Surg, 2010. 8(5): p. 336-341.

14. Higgins, J.P. and S.G. Thompson, Quantifying heterogeneity in a meta-analysis. Statistics in medicine, 2002. 21(11): p. 1539-1558.

15. George, B.J. and I.B. Aban, An application of meta-analysis based on DerSimonian and Laird method. 2016, Springer.

16. IntHout, J., J.P. Ioannidis, and G.F. Borm, The Hartung-Knapp-Sidik-Jonkman method for random effects meta-analysis is straightforward and considerably outperforms the standard DerSimonianLaird method. BMC medical research methodology, 2014. 14(1): p. 25.

17. https://www.rcsb.org/structure/6LU7.

18. Trott, O. and A.J. Olson, AutoDock Vina: improving the speed and accuracy of docking with a new scoring function, efficient optimization, and multithreading. Journal of computational chemistry, 2010. 31(2): p. 455-461. 
19. Barnard, D.L., et al., Is the anti-psychotic, 10-(3-(dimethylamino) propyl) phenothiazine (promazine), a potential drug with which to treat SARS infections?: Lack of efficacy of promazine on SARS-CoV replication in a mouse model. Antiviral research, 2008. 79(2): p. 105-113.

20. Day, C.W., et al., A new mouse-adapted strain of SARS-CoV as a lethal model for evaluating antiviral agents in vitro and in vivo. Virology, 2009. 395(2): p. 210-222.

21. Barnard, D.L., et al., Evaluation of immunomodulators, interferons and known in vitro SARS-coV inhibitors for inhibition of SARS-coV replication in BALB/c mice. Antiviral Chemistry and Chemotherapy, 2006. 17(5): p. 275-284.

22. Barnard, D.L., et al., Enhancement of the infectivity of SARS-COV in BALB/c mice by IMP dehydrogenase inhibitors, including ribavirin. Antiviral research, 2006. 71(1): p. 53-63.

23. Sheahan, T.P., et al., An orally bioavailable broad-spectrum antiviral inhibits SARS-CoV-2 in human airway epithelial cell cultures and multiple coronaviruses in mice. Science translational medicine, 2020.

24. Sheahan, T.P., et al., Broad-spectrum antiviral GS-5734 inhibits both epidemic and zoonotic coronaviruses. Science translational medicine, 2017. 9(396).

25. Totura, A.L. and R.S. Baric, SARS coronavirus pathogenesis: host innate immune responses and viral antagonism of interferon. Current opinion in virology, 2012. 2(3): p. 264-275.

26. Van Reeth, K., S. Van Gucht, and M. Pensaert, In vivo studies on cytokine involvement during acute viral respiratory disease of swine: troublesome but rewarding. Veterinary immunology and immunopathology, 2002. 87(3-4): p. 161-168.

27. Cheung, C., et al., Induction of proinflammatory cytokines in human macrophages by influenza $A$ (H5N1) viruses: a mechanism for the unusual severity of human disease? The Lancet, 2002. 360(9348): p. 1831-1837.

28. Mogensen, T.H. and S.R. Paludan, Molecular pathways in virus-induced cytokine production. Microbiol. Mol. Biol. Rev., 2001. 65(1): p. 131-150.

29. Wong, C., et al., Plasma inflammatory cytokines and chemokines in severe acute respiratory syndrome. Clinical \& Experimental Immunology, 2004. 136(1): p. 95-103.

30. Lalu, M.M., et al., Identifying stroke therapeutics from preclinical models: A protocol for a novel application of network meta-analysis. F1000Research, 2019. 8.

31. Xia, B. and X. Kang, Activation and maturation of SARS-CoV main protease. Protein \& cell, 2011. 2(4): p. 282-290.

32. Lu, I.-L., et al., Structure-based drug design and structural biology study of novel nonpeptide inhibitors of severe acute respiratory syndrome coronavirus main protease. Journal of medicinal chemistry, 2006. 49(17): p. 5154-5161.

33. Kumar, Y. and $\mathrm{H}$. Singh, In silico identification and docking-based drug repurposing against the main protease of SARS-CoV-2, causative agent of COVID-19. 2020. 

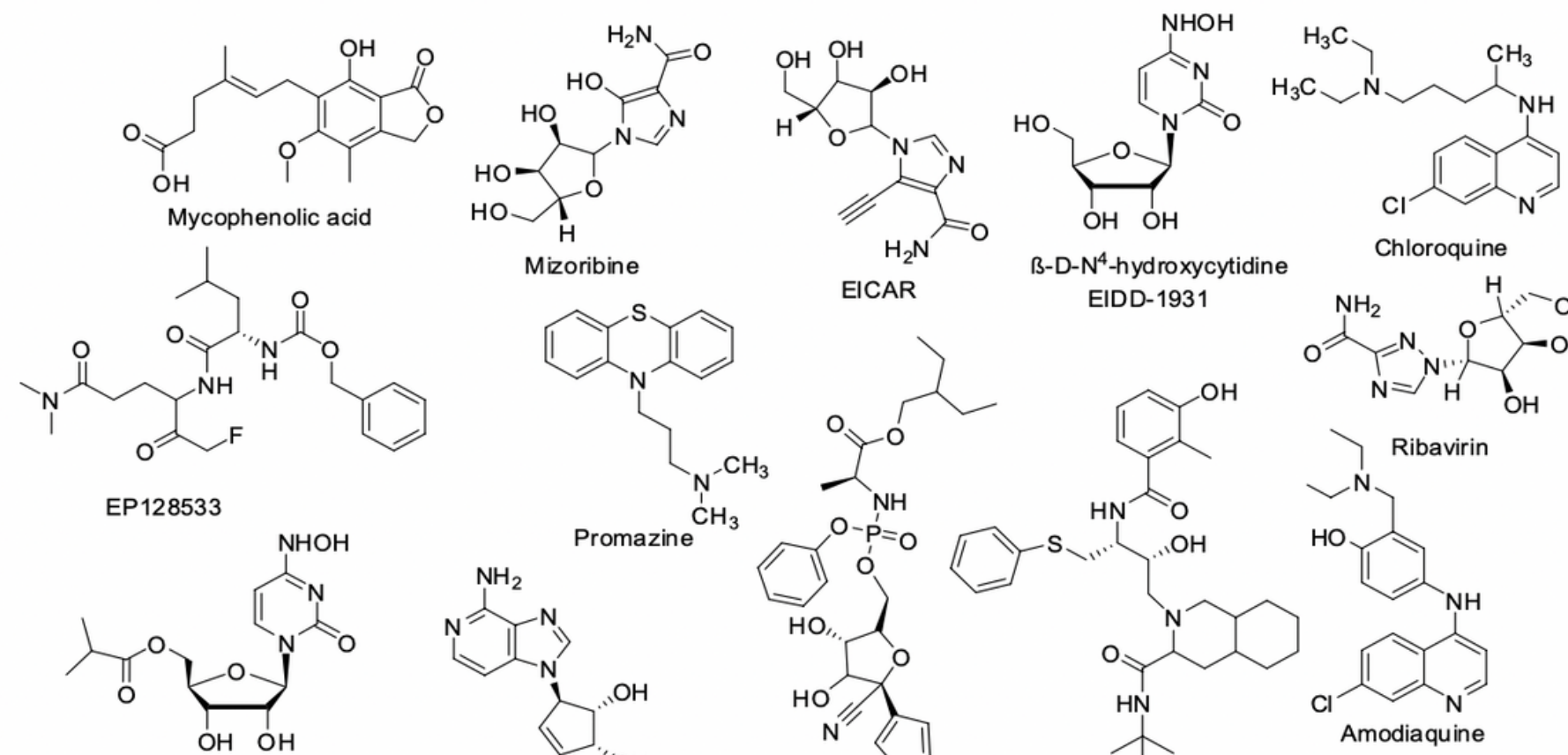

B-D-N $N^{4}$-hydroxycytidine-5'-isopropyl ester EIDD-2801

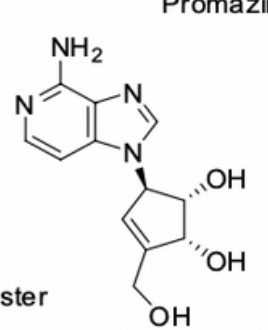

3-Deazaneplanocin

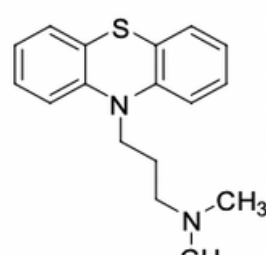

$\mathrm{CH}_{3}$<smiles>c1ccccc1</smiles><smiles>COP(N)O</smiles><smiles>CCSc1ccccc1</smiles>

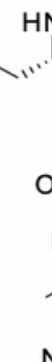

$$
\text { EIDD-1931 }
$$

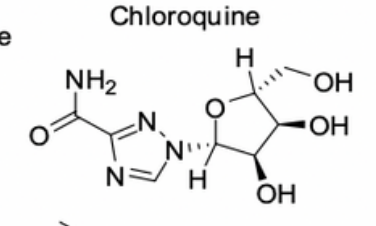

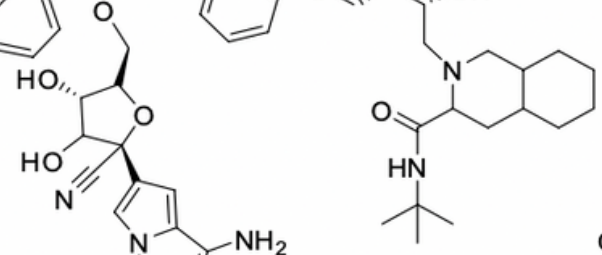

Remdesivir (GS5734)<smiles>Cc1c(O)cccc1C(N)=O</smiles><smiles>CCN(CC)CC</smiles>

$\mathrm{H}$<smiles>CCC=CNc1ccnc2ccc(Cl)cc12</smiles>
Amodiaquine<smiles>CC(=O)CCCCn1c(=O)c2c(ncn2C)n(C)c1=O</smiles>

Pentoxifylline

\section{Figure 1}

Structures of compounds evaluated for efficacy of SARS-CoV in the animal models. 


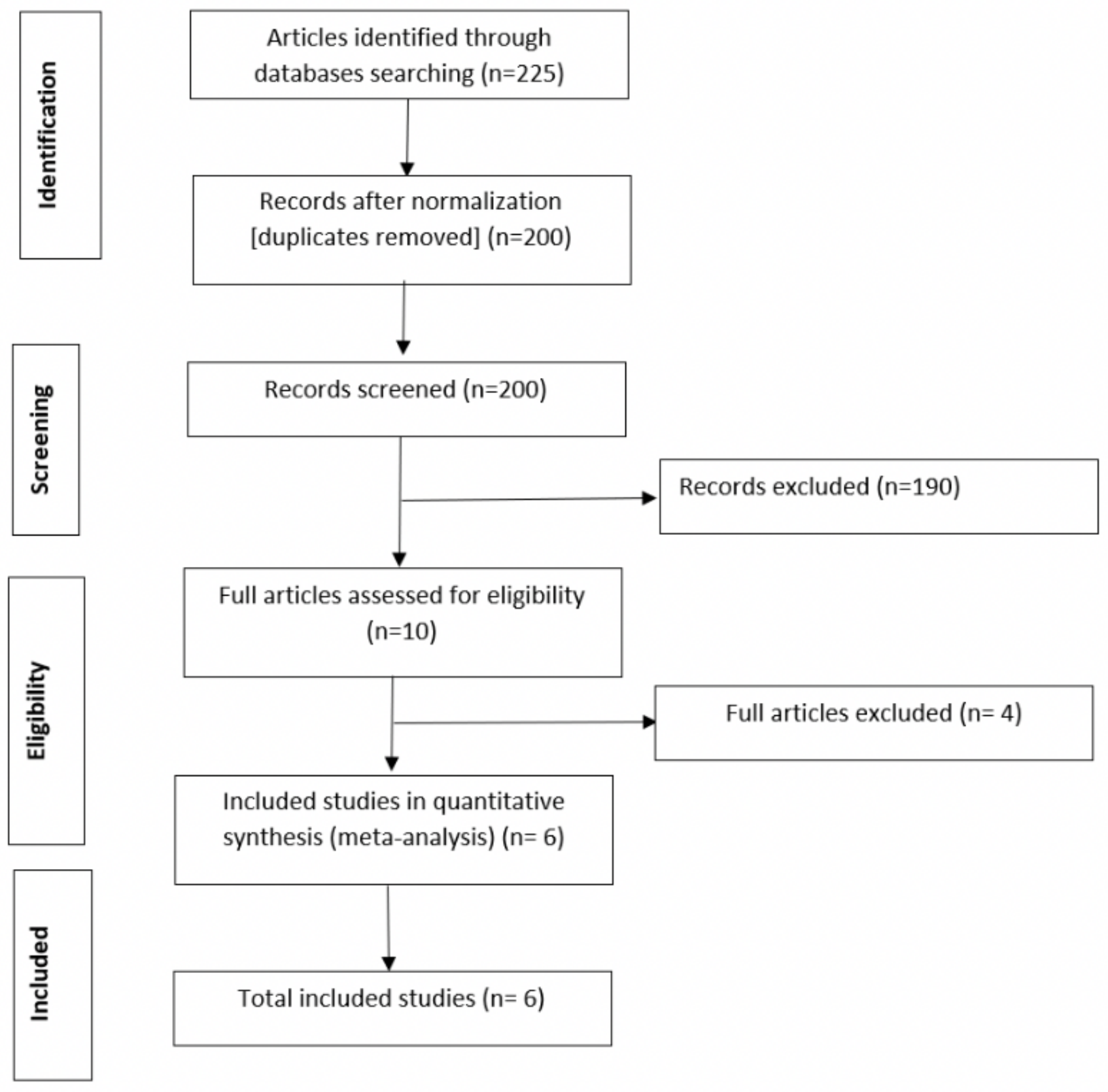

Figure 2

Flow diagram of the database searches 


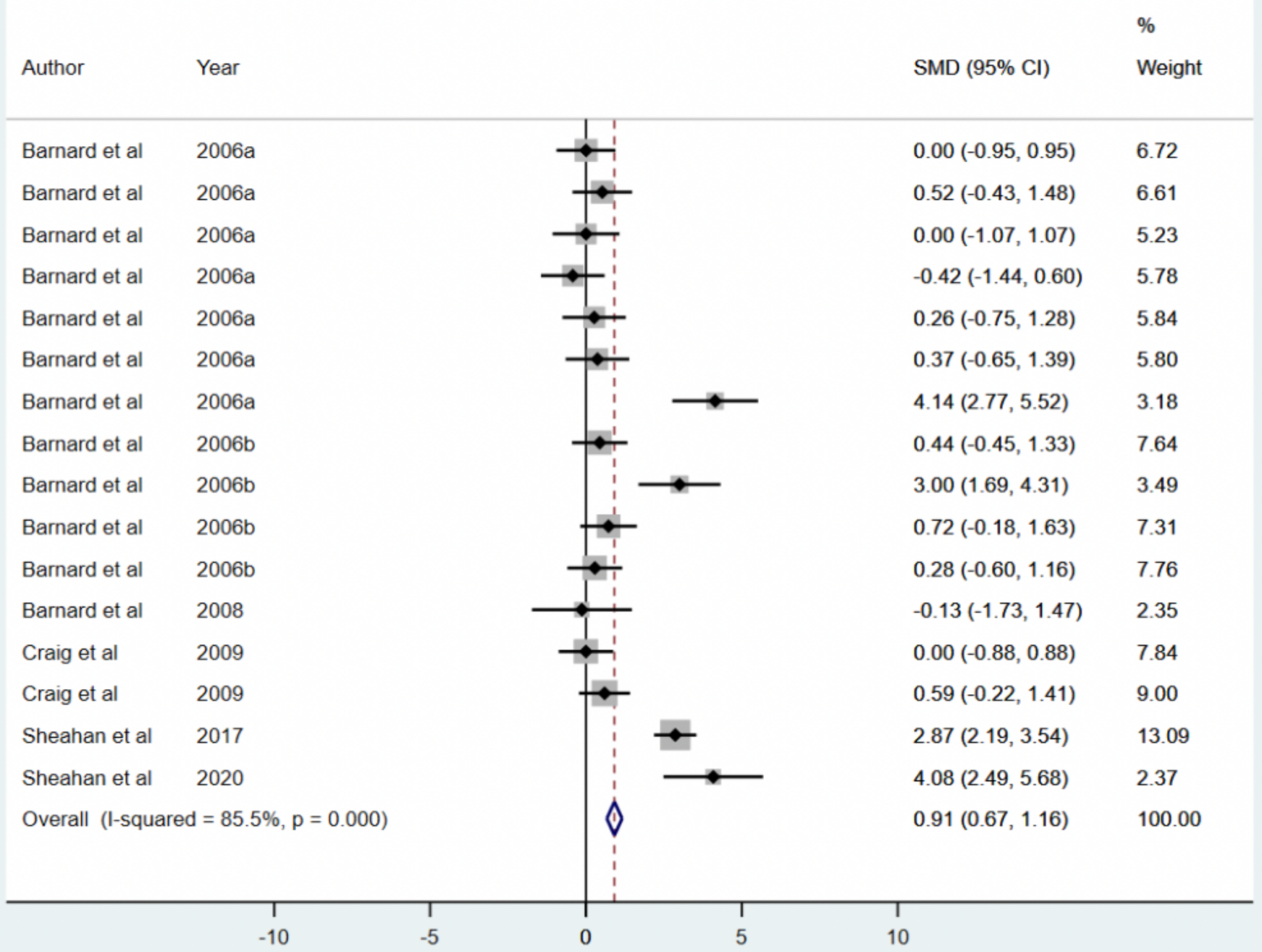

\section{Figure 3}

Forest plot obtained from meta-analysis comparing the mean difference of virus titer in infected mice treated with the selected compounds and those untreated (control). 
Study

ID

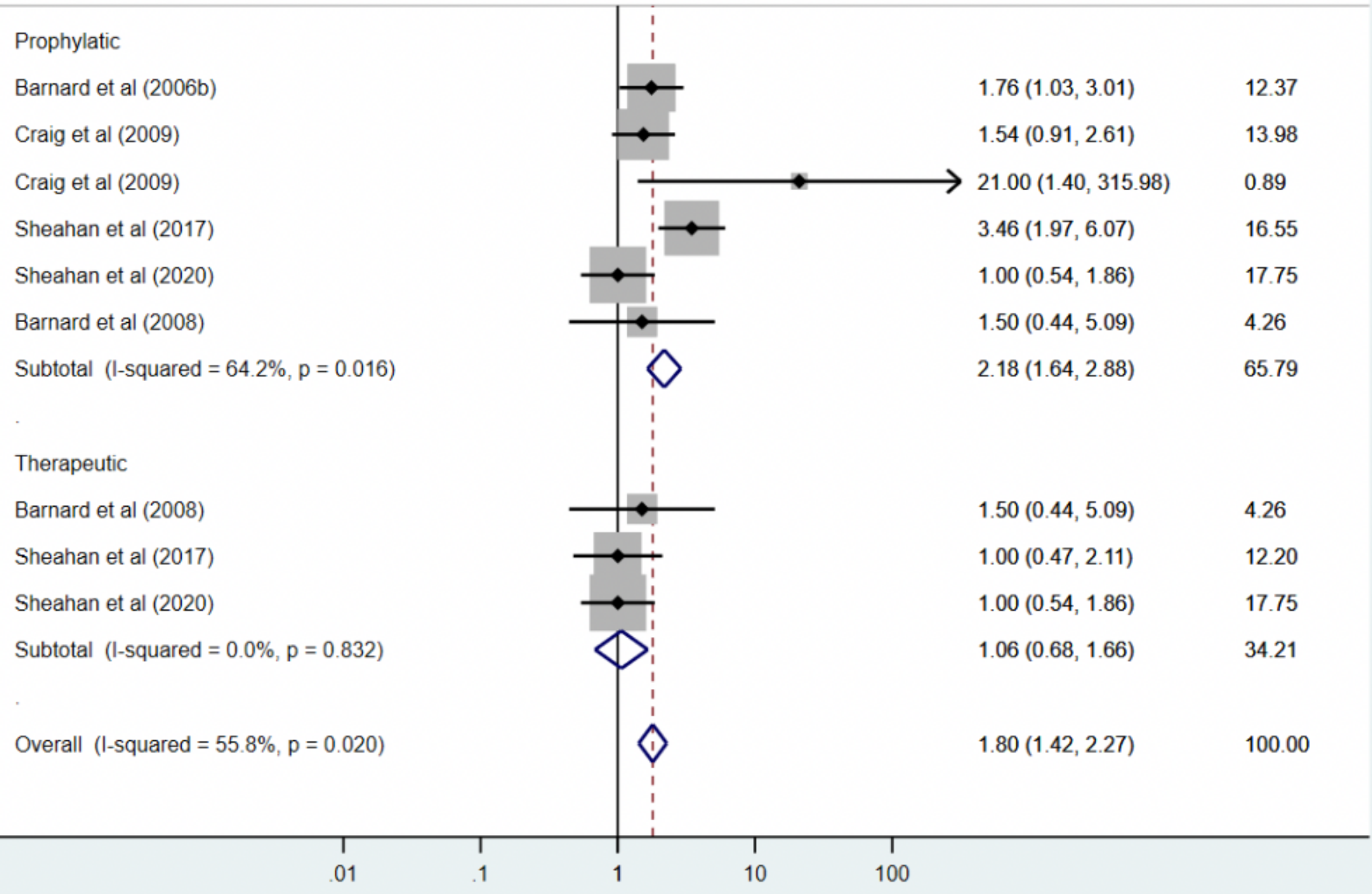

\section{Figure 4}

Forest plot obtained from meta-analysis comparing the mortality in infected mice treated with the selected compounds. 


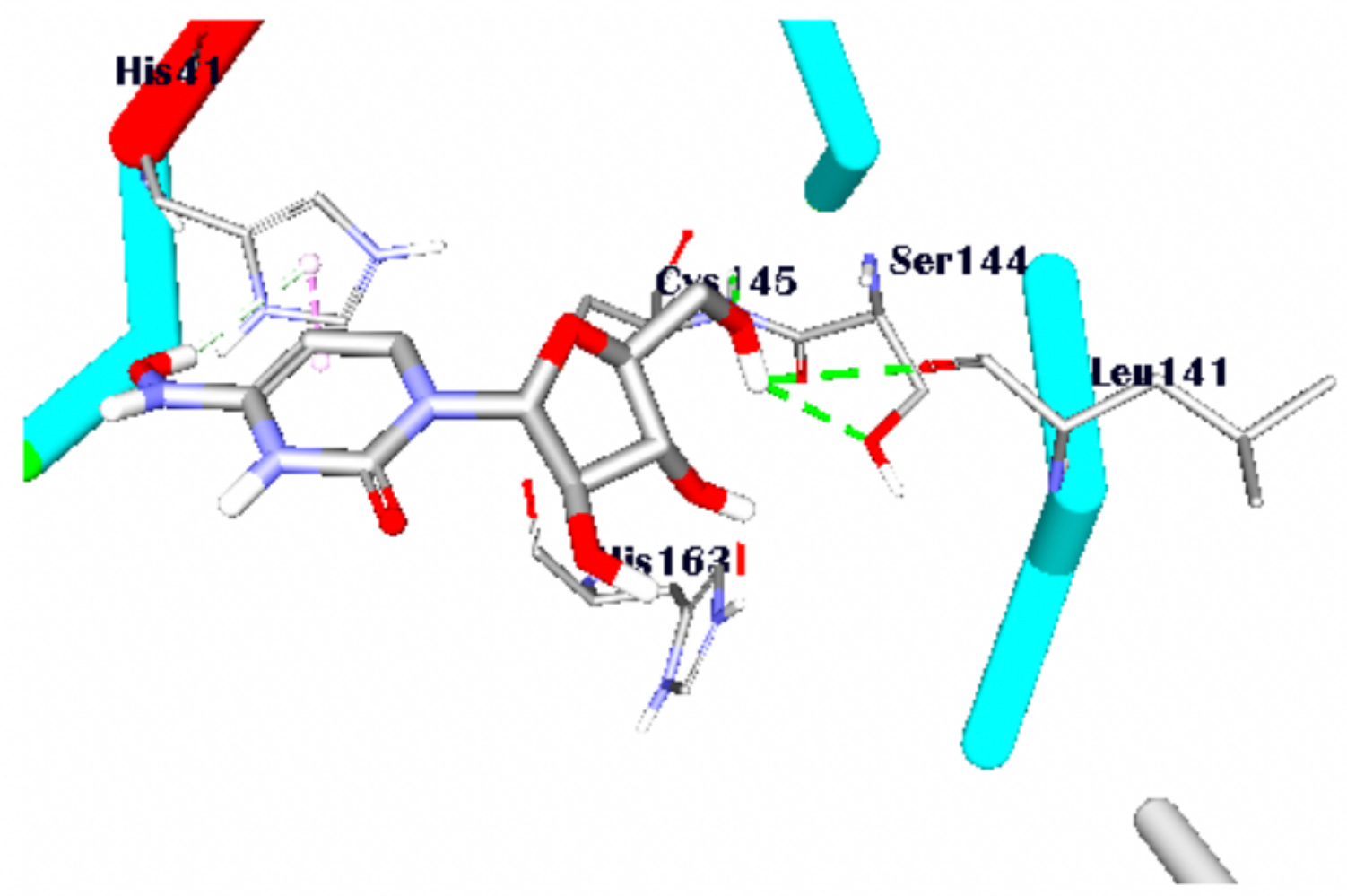

Figure 5. Schematic representation of COVID-19 main protease (PDB: 6LU7) interactions with EIDD-1931. = protein ligand O hydrophobic interaction Ohydrogen bond

Figure 5 


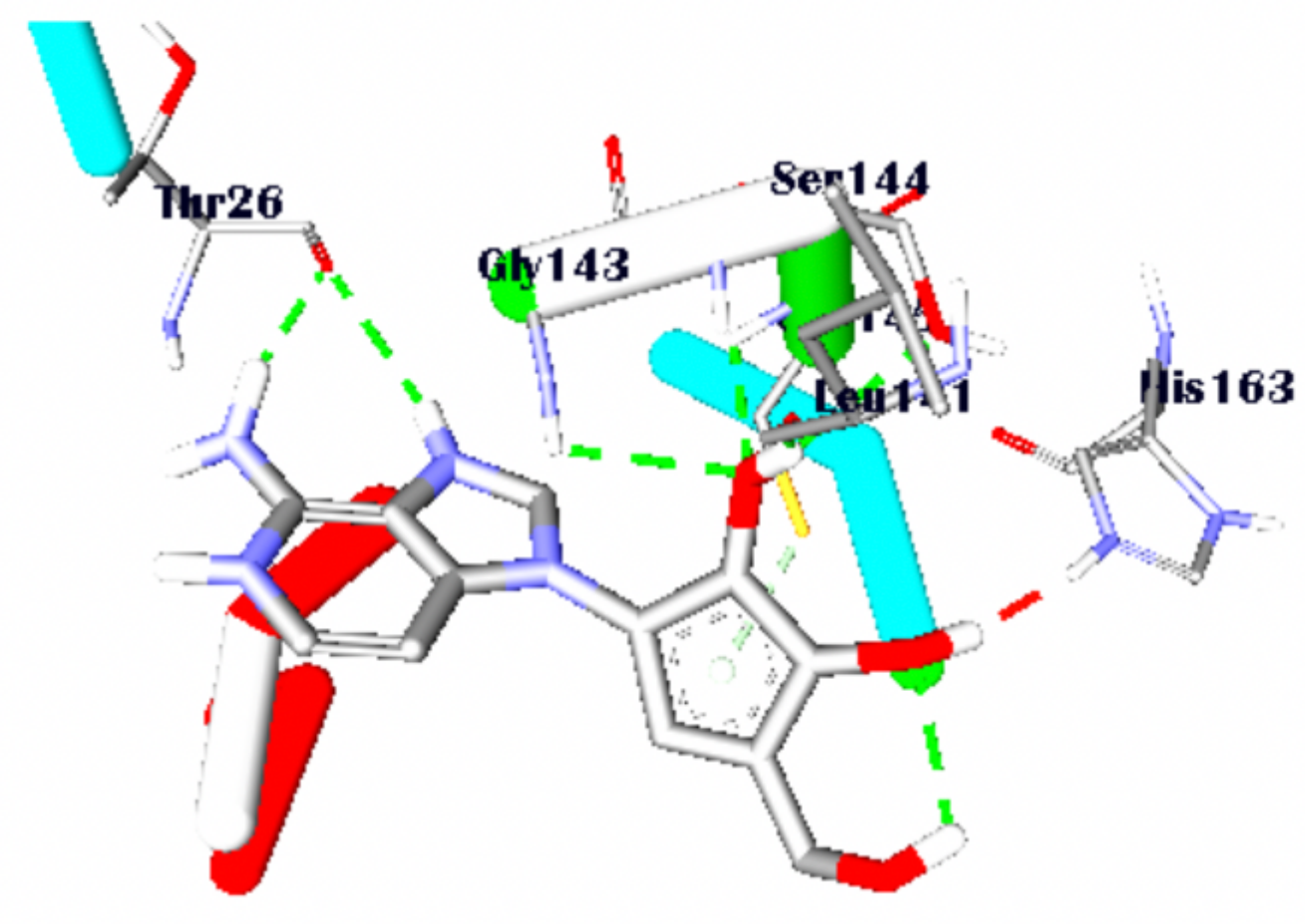

Figure 6. Schematic representation of COVID-19 main protease (PDB: 6LU7) interactions with 3-Deazaneplanocin. $=$ protein " ligand $\mathrm{O}$ hydrophobic interaction Ohydrogen bond

Figure 6 


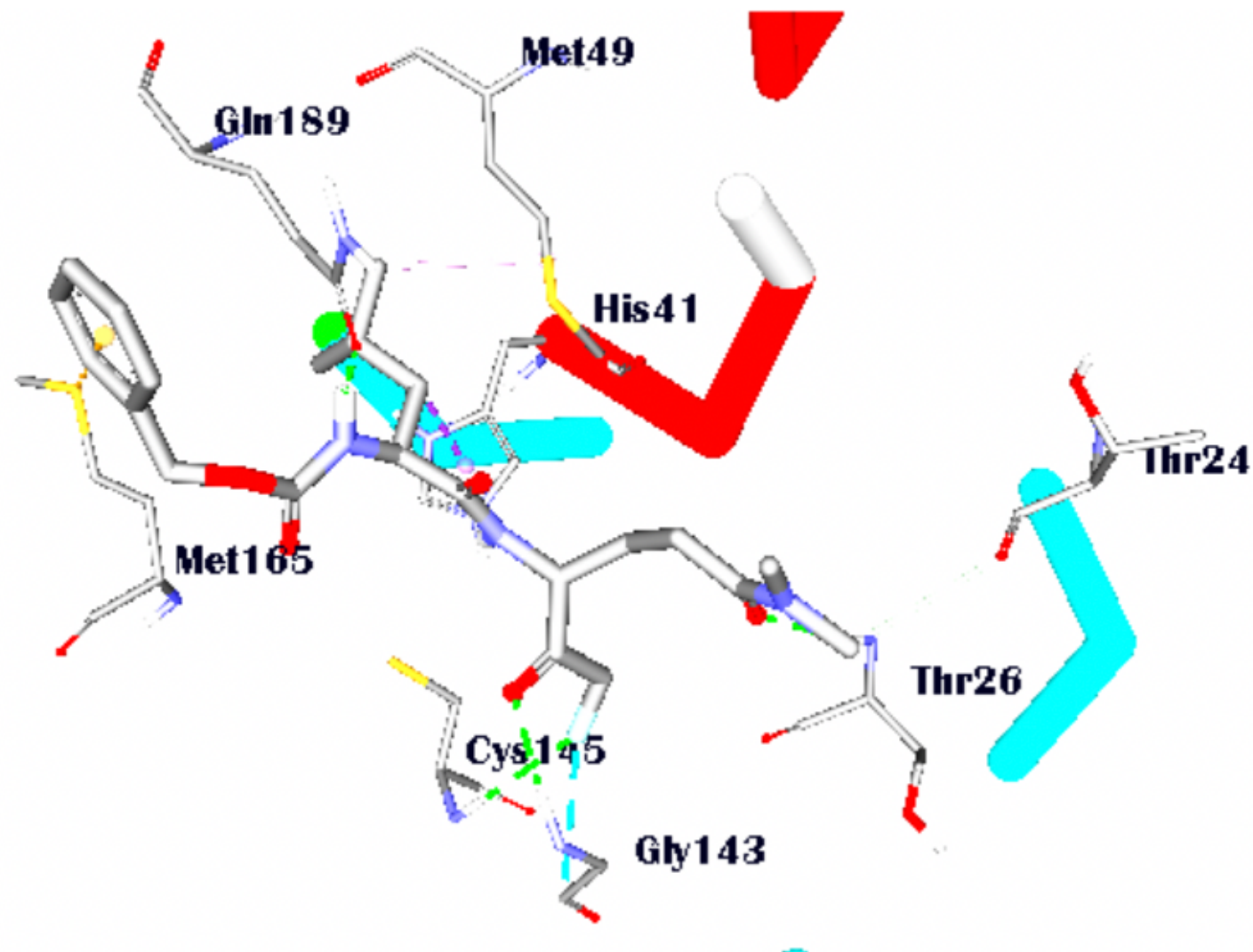

Figure 7. Schematic representation of COVID-19 main protease (PDB: 6LU7) interactions with EP128533. = protein $=$ ligand Ohydrophobic interaction O hydrogen bond

Figure 7 


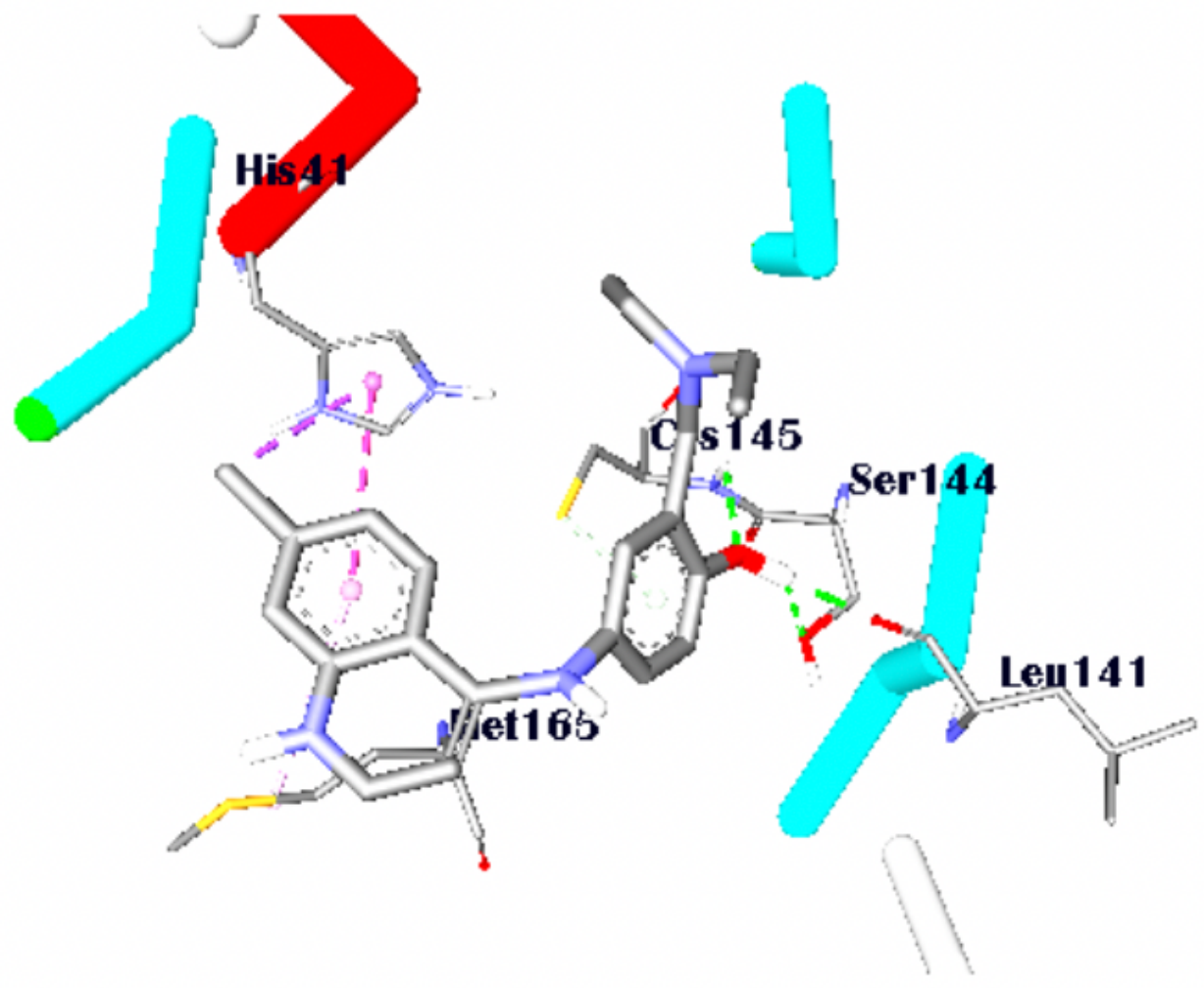

Figure 8. Schematic representation of COVID-19 main protease (PDB: 6LU7) interactions with amodiaquine. $=$ protein $=$ ligand ohydrophobic interaction $\mathrm{O}$ hydrogen bond

Figure 8 


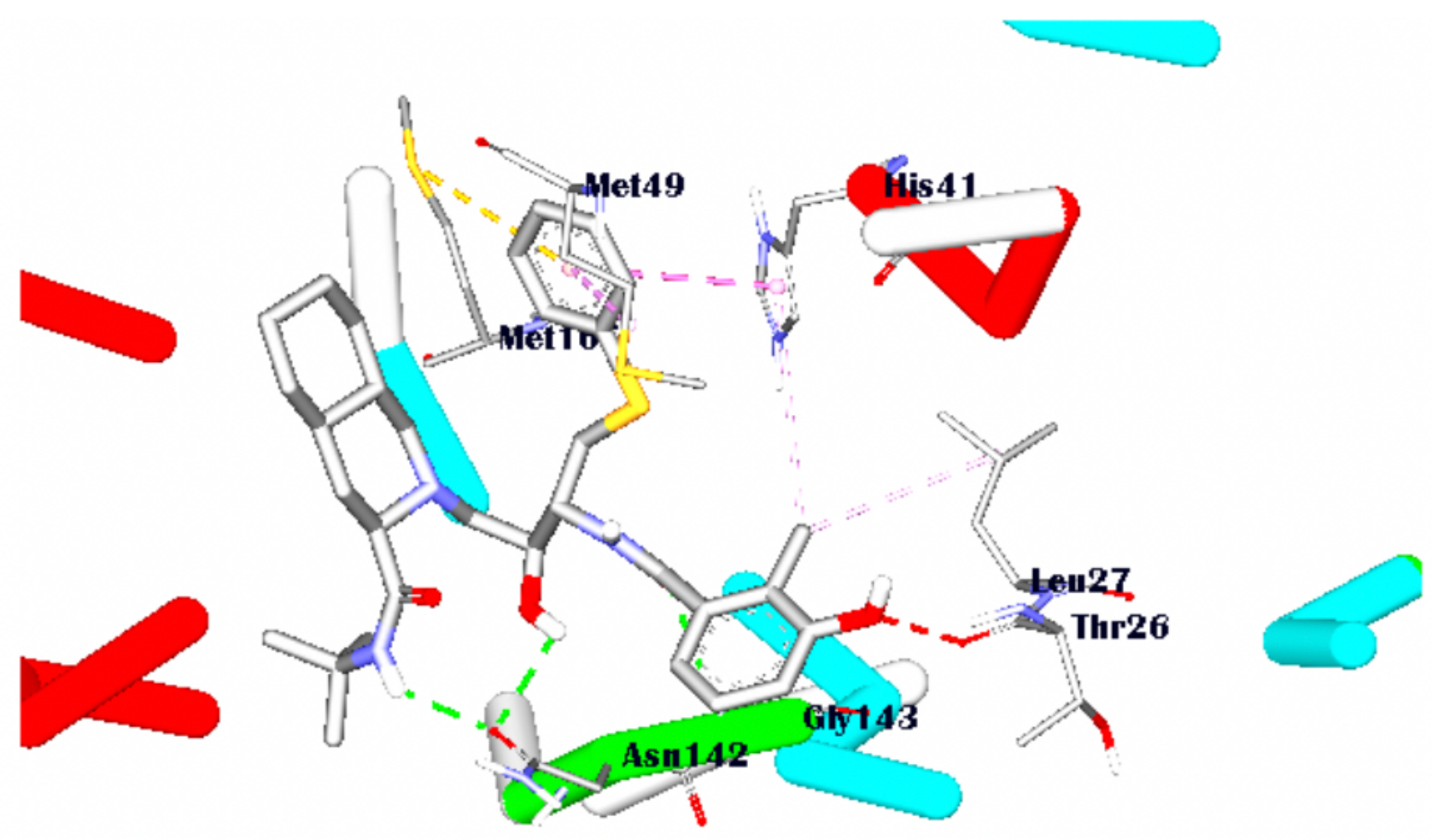

Figure 9. Schematic representation of COVID-19 main protease (PDB: 6LU7) interactions with nelfinavir. $=$ protein ligand Ohydrophobic interaction Ohydrogen bond

Figure 9 

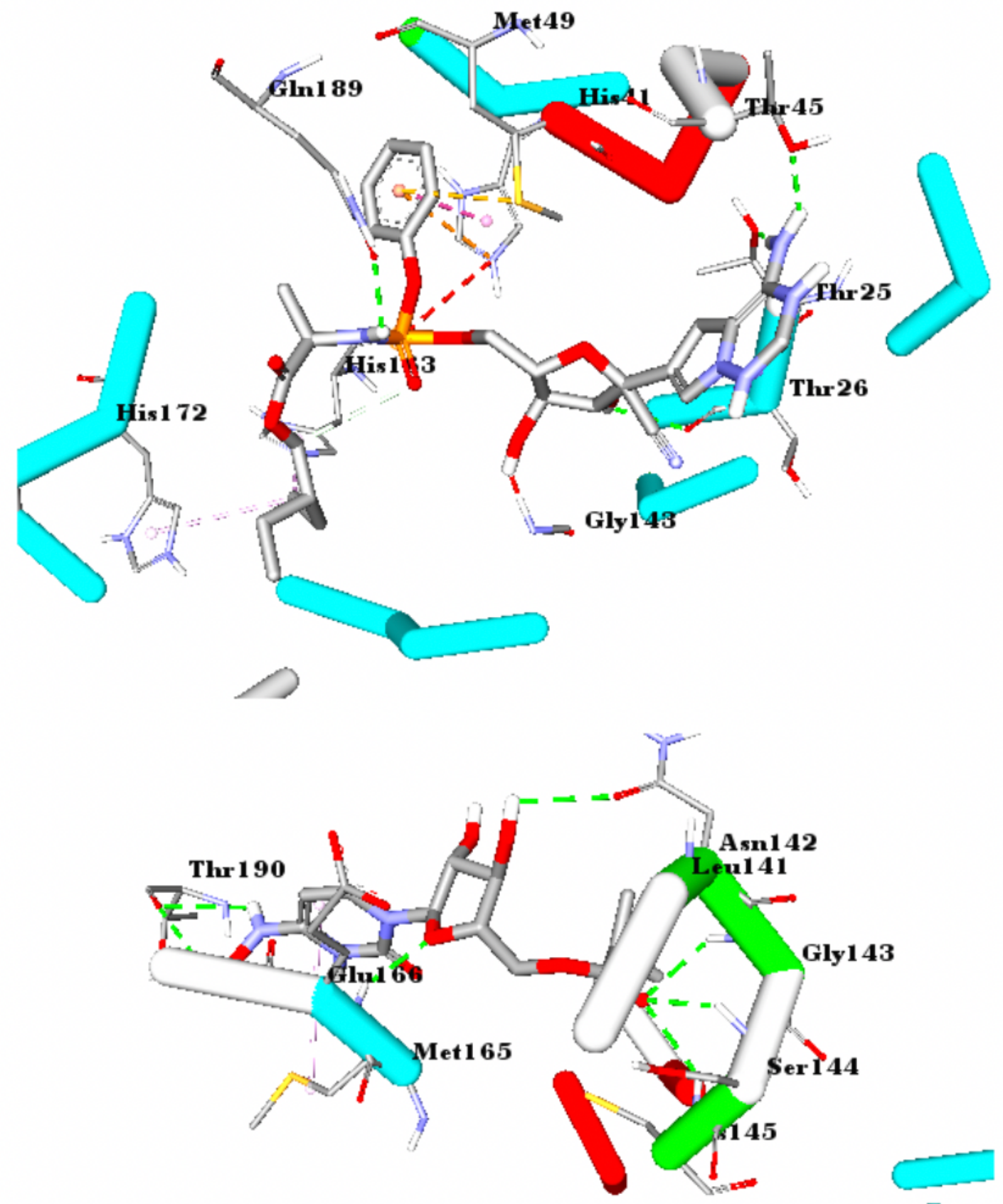

Figure 10. Schematic representation of COVID-19 main protease (PDB: 6LU7) interactions with remdesivix \& EIDD-2801 " protein $=$ ligand $\bigcirc$ hydrophobic interaction $\bigcirc$ hydrogen bond

Figure 10

\section{Supplementary Files}

This is a list of supplementary files associated with this preprint. Click to download.

- SuppinfoPaper2DataANALYSED.xlsx 